\title{
Epilepsia en niños atendidos en el Hospital Nacional Cayetano Heredia de Lima, Perú, 2010- 2016.
}

Clinical characteristics of epileptic children seen at Cayetano Heredia Hospital. Lima, Perú, 2010-2016.

\begin{abstract}
Daniel Guillén - Pinto 1,2,a,b, Catherine V. Gonzales ${ }^{1, c}$, Werner Vidal ${ }^{1, c}$, Carla Santivañez ${ }^{1, \mathrm{c}}$, Judith Vila ${ }^{1,2, a, b}$, Tania Juárez 1,b, Julissa Guevara ${ }^{1, b}$, Elizabeth Espíritu ${ }^{1, b}$, Rosa Alvarado ${ }^{1, b}$, Cynthia Samaniego ${ }^{1,2, d}$, Susan Samalvides ${ }^{1, b}$, Luis M. Milla ${ }^{1, b}$, Iván O. Espinoza ${ }^{1,2, a, b}$
\end{abstract}

\section{RESUMEN}

La epilepsia es la principal causa de consulta neurológica en niños peruanos. Sin embargo, hay escasa información sobre sus características clínicas y epidemiológicas. Objetivos: Presentar las características clínicas de los niños epilépticos atendidos en el Hospital Cayetano Heredia, entre los años 2010 y 2016. Material y métodos: Se realizó un estudio observacional descriptivo, retrospectivo, tipo serie de casos, basado en las historias clìnicas de en niños menores de 14 años con diagnóstico de epilepsia, seguimiento clínico, registro electroencefalogràfico y neuroimagenes. Resultados: Se incluyeron 193 pacientes. El 47,2\% (91/193) presentaron su primera crisis epiléptica antes del año de edad, cuya etiología fue secundaria en el 59,3\% (54/91), destacando los factores perinatales y las malformaciones cerebrales. La etiología primaria fue más frecuente en los niños que iniciaron las crisis después del año de edad. Las crisis generalizadas se presentaron en 64,2\% (124/193) sin diferencias por grupos etarios. El 69,9\% (135/193) fueron controlados con un solo fármaco, en tanto que el 15\% (29/193) fueron refractarios al tratamiento. La comorbilidad ocurrió en el 68,4\% (132/193), siendo frecuentes el retraso del desarrollo psicomotor, el retardo mental y la parálisis cerebral. Conclusiones: La mayoría de los niños presentaron su primera crisis antes del año de edad, generalmente de causa secundaria. En los niños mayores fue prevalente la etiología primaria. La mayoría fueron controlados con monoterapia, pero se registró un porcentaje de refractariedad al tratamiento. La comorbilidad fue muy frecuente.

PALABRAS CLAVE: Epilepsia, niños, tratamiento, comorbilidad, etiología, Perú.

\section{SUMMARY}

Epilepsy is the main cause of neurological clinical visits in Peruvian children. However, in our country, there is little information on its clinical and epidemiological characterizes. Objectives: To describe the clinical characteristics of children with epilepsy seen at the Cayetano Heredia Hospital, between 2010 and 2016. Material and Methods: A descriptive retrospective observational study was carried out, based on the clinical records of children under 14 years old with diagnosis of epilepsy, with clinical follow-up, electroencephalography and neuroimaging. Results: One hundred ninety-three patients were included. In 47.2\% (91/193), the first seizure occurred before the one year of age; the etiology was secondary in 59.3\% (54/91), with the main causes being perinatal events and cerebral malformations. Primary etiologies were more frequent in the children who started haviing seizures after one year of age. Generalized seizures ocurred in $64.2 \%$ (124/193) without differences by age groups. In 69.9\% (135/193) seizures were controlled with a single drug while $15 \%$ (29/193) were refractory to treatment. Comorbidity occurred in

\footnotetext{
${ }^{1}$ Hospital Nacional Cayetano Heredia. Lima, Perú.

${ }^{2}$ Universidad Peruana Cayetano Heredia. Lima, Perú.

${ }^{\text {a }}$ Docente; ${ }^{\mathrm{b}}$ Neurólogo Pediatra; ${ }^{\mathrm{c}}$ Estudiante; ${ }^{\mathrm{d}}$ Residente
} 
68,4\% (132/193), with delays in psychomotor development, intellectual disability and cerebral palsy being frequent. Conclusions: Most children had their first seizures before the first year of age, usually with a secondary cause. In older children, the primary epilepsies were more prevalent. Monotherapy controlled the majority of children, but a percentage of refractoriness to the treatment was registered. Comorbidities were very frequent.

KEY WORDS: Epilepsy, children, treatment, comorbidity, etiology, Peru.

\section{INTRODUCCIÓN}

La epilepsia incluye diferentes tipos de enfermedades caracterizadas por la recurrencia de crisis epilépticas, tanto primarias como secundarias $(1,2)$. En países desarrollados la prevalencia es de 3-4 x 1000 habitantes, comparado con 5-77 x 1000 habitantes en países en desarrollo, esta prevalencia correlaciona con la mayor frecuencia de algunos factores de riesgos, factores genéticos, metodológicos, entre otros $(3,4)$. En niños, la prevalencia varía de 3.4-8 x 1000 habitantes dependiendo del nivel de desarrollo del país $(5,6,7)$.

La epilepsia tiene formas clínicas de acuerdo a la edad del niño, que son de importancia para el diagnóstico y manejo. Y que, en ocasiones conforman verdaderos patrones clínico electroencefalográficos (8). En ese sentido, la clasificación del tipo de epilepsia ayuda a determinar el diagnóstico y pronóstico de la enfermedad (9).

Como formas primarias destacan las epilepsias neonatales, las encefalopatías epilépticas, las epilepsias benignas, las epilepsias mioclónicas, las epilepsias focales y las epilepsias ausencias. Como formas secundarias, las epilepsias derivadas de los trastornos metabólicos, traumáticos, tóxicos e infecciosos son las más frecuentes $(4,8)$. En cualquier caso, la mayor recurrencia y/o excesiva duración de las crisis se asocian con problemas cognitivos, conductuales y estructurales del sistema nervioso (10).

Motivados por la insuficiente información de los establecimientos peruanos de salud, se realizó el presente trabajo cuyo objetivo fue presentar las características clínicas de los niños epilépticos atendidos en el Hospital Cayetano Heredia, entre los años 2010 y 2016.

\section{MATERIAL Y MÉTODOS}

Se realizó un estudio observacional descriptivo, retrospectivo, tipo serie de casos, cuya fuente fue la base de datos del Servicio de Neuropediatría y el Archivo de la Oficina de Estadística del Hospital Nacional Cayetano Heredia (HNCH), en Lima-Perú. El HNCH es un establecimiento de salud del tercer nivel que atiende una población urbano marginal.

La población fue seleccionada de acuerdo con los siguientes criterios: Pacientes menores de 14 años con diagnóstico de epilepsia, atendidos entre los años 2010-2016 en la consulta externa del Servicio de Neuropediatría, los cuales contaban con electroencefalograma (EEG), tomografía computarizada (TC) o resonancia magnética (RM) y seguimiento neurológico mínimo dos veces por año en el servicio mencionado. Fueron excluidos los pacientes con enfermedades regresivas y con datos incompletos.

Todos los casos fueron diagnosticados por un neuropediatra. Para la etiología de las crisis y los tipos de crisis epilépticas se utilizaron las definiciones establecidas por la ILAE 1989, usadas durante este periodo en esta institución (11). Idiopáticas, sin causa identificable, criptogénicas cuando se sospecha una causa pero no se pudo identificar y secundaria cuando se identifica el origen. La condición de refractariedad (epilepsia resistente a fármacos) fue definida como la falta de control de crisis habiendo usado dos medicamentos a dosis máximas por 6 meses (3).

Las causas secundarias se clasificaron en malformaciones cerebrales (esquizencefalia, liscencefalia, otros), eventos perinatales (encefalopatía hipóxico- isquémica, asfixia neonatal, hemorragia intraventricular, otros), infecciones (meningoencefalitis, encefalitis), cerebrovasculares (accidentes cerebrovasculares), síndromes neurocutáneos (esclerosis tuberosa, síndrome Sturge Weber, neurofibromatosis), toxinas (intoxicación por piretroides o anís estrella), traumatismo encefalocraneano y esclerosis mesial temporal.

La información fue almacenada en una base de datos electrónica y la identidad de los pacientes se protegió mediante códigos numéricos. Asimismo, el proyecto fue aprobado por el Comité Institucional de 
Ética de la Universidad Peruana Cayetano Heredia y el Comité de Ética del Hospital Nacional Cayetano Heredia.

El análisis estadístico se realizó calculando frecuencias de cada variable, para lo cual se utilizó el paquete estadístico SPSS (IBM SPSS Statistics para Windows versión 21.0 Armonk, NY; IBM Corp) y el programa Microsoft Word 2013.

\section{RESULTADOS}

Se identificaron 236 pacientes en la base de datos, de los cuales se excluyeron 43 por tener datos incompletos (tipo de crisis, edad de primera crisis y neuroimagen), de éstos $60,5 \%$ (26/43) fueron de causa secundaria y $23,2 \%(10 / 43)$ de causa primaria. Cuatro de los niños con epilepsia primaria se excluyeron por no tener neuroimagen. Más de la mitad de los que fueron excluidos tuvieron falta múltiple de datos, entre ellos, se encontró un caso de neurocisticercosis. Finalmente, fueron seleccionados 193 pacientes.

La población de estudio tuvo una razón varón/mujer de 1,2:1. La mayoría de los pacientes procedieron de Lima y Callao en $84,9 \%$. La mediana de edad para la primera crisis epiléptica fue de un año.

Tabla 1. Características clínicas de los niños epilépticos atendidos en el Hospital Cayetano Heredia. Lima, Perú 2010-2016.

\begin{tabular}{|c|c|c|}
\hline Características & $\mathbf{n}$ & $\%$ \\
\hline \multicolumn{3}{|l|}{ Sexo } \\
\hline Masculino & 104 & 53,9 \\
\hline Femenino & 89 & 46,1 \\
\hline \multicolumn{3}{|l|}{ Procedencia } \\
\hline Lima norte & 135 & 70,0 \\
\hline Lima otros distritos & 35 & 18,1 \\
\hline Otras regiones & 11 & 5,7 \\
\hline No determinado & 12 & 6,2 \\
\hline \multicolumn{3}{|l|}{ Edad de primera crisis (años) } \\
\hline$<1$ & 91 & 47,2 \\
\hline $1-5$ & 63 & 32,6 \\
\hline 6-14 & 39 & 20,2 \\
\hline \multicolumn{3}{|l|}{ Tipo de Crisis } \\
\hline Focal & 69 & 35,8 \\
\hline Generalizada & 124 & 64,2 \\
\hline \multicolumn{3}{|l|}{ Etiología } \\
\hline Primaria & 71 & 36,8 \\
\hline Secundaria & 89 & 46,1 \\
\hline Criptogénica & 33 & 17,1 \\
\hline \multicolumn{3}{|l|}{ Tratamiento } \\
\hline 1 fármaco & 135 & 69,9 \\
\hline 2 fármacos & 44 & 22,8 \\
\hline$\geq 3$ fármacos & 14 & 7,3 \\
\hline \multicolumn{3}{|l|}{ Epilepsia Refractaria } \\
\hline $\mathrm{Si}$ & 29 & 15,0 \\
\hline No & 164 & 85,0 \\
\hline \multicolumn{3}{|l|}{ Comorbilidad } \\
\hline 1 & 69 & 35,8 \\
\hline 2 & 51 & 26,4 \\
\hline$\geq 3$ & 12 & 6,2 \\
\hline
\end{tabular}


Tabla 2. Etiología de la epilepsia según edad de primera crisis en los niños epilépticos atendidos en el Hospital Cayetano Heredia. Lima, Perú 2010-2016.

\begin{tabular}{|c|c|c|c|c|c|c|c|c|}
\hline \multirow{2}{*}{ Etiología } & \multicolumn{2}{|c|}{ Niños } & \multicolumn{2}{|c|}{$<1$ año } & \multicolumn{2}{|c|}{ 1-5 años } & \multicolumn{2}{|c|}{ 6-14 años } \\
\hline & $\mathbf{n}$ & $\%$ & $\mathbf{n}$ & $\%$ & $\mathbf{n}$ & $\%$ & $\mathbf{n}$ & $\%$ \\
\hline Primaria & 71 & 36,8 & 24 & 26,4 & 33 & 44,6 & 14 & 50,0 \\
\hline Criptogénica & 33 & 17,1 & 13 & 14,3 & 15 & 20,3 & 5 & 17,9 \\
\hline Secundaria & 89 & 46,1 & 54 & 59,3 & 26 & 35,1 & 9 & 32,1 \\
\hline Malformación cerebral & 21 & 10,9 & 12 & 13,2 & 5 & 6,7 & 4 & 14,2 \\
\hline Eventos perinatales & 14 & 7,3 & 13 & 14,3 & 1 & 1,3 & 0 & 0,0 \\
\hline Infección del SNC & 13 & 6,7 & 11 & 12,0 & 2 & 2,7 & 0 & 0,0 \\
\hline Cerebrovasculares & 11 & 5,7 & 9 & 9,9 & 0 & 0,0 & 2 & 7,1 \\
\hline Síndromes neurocutáneos & 7 & 3,6 & 3 & 3,3 & 4 & 5,4 & 0 & 0,0 \\
\hline Esclerosis mesial temporal & 7 & 3,6 & 3 & 3,3 & 3 & 4,1 & 1 & 3,6 \\
\hline Toxinas & 7 & 3,6 & 1 & 1,1 & 5 & 6,7 & 1 & 3,6 \\
\hline Traumatismo encefalocraneano & 5 & 2,6 & 1 & 1,1 & 3 & 4,1 & 1 & 3,6 \\
\hline Otros & 4 & 2,1 & 1 & 1,1 & 3 & 4,1 & 0 & 0,0 \\
\hline Total & 193 & & 91 & & 74 & & 28 & \\
\hline
\end{tabular}

El 47,2\% (91/193) de los niños eran menores de un año de edad cuando iniciaron la enfermedad. La etiología secundaria fue la más frecuente en $46,1 \%$ (89/193) y se observó que mientras la etiología secundaria fue disminuyendo con la edad, la etiología primaria fue aumentando (tabla 1). En los niños que iniciaron la enfermedad antes del año de edad, las causas secundarias alcanzaron el 59,3\% (54/91) (tabla 2).

La etiología secundaria fue muy variable dependiendo del grupo etario. En los menores de un año predominaron los eventos perinatales, $24,1 \%(13 / 54)$, las malformaciones $22,2 \%(12 / 54)$ y las infecciones del SNC 20,4 \% (11/54). En los mayores de un año, se observó una miscelánea, sin predominio entre malformaciones, infecciones, tóxicos y síndromes neurocutáneos (tabla 2).

Las crisis generalizadas se presentaron en $64,2 \%$ (124/193) de los pacientes; de las cuales 42,7\% (53/124) fueron tónico clónicas y 20,9\% (26/124) tónicas. Las crisis atónicas fueron excepcionales alcanzando solo el 4\% (5/124) de este grupo. Por otro lado, el 35,8

Tabla 3. Etiología de la epilepsia según el tipo de crisis en los niños epilépticos atendidos en el Hospital Cayetano Heredia. Lima, Perú 2010-2016.

\begin{tabular}{lccccccc}
\hline \multirow{2}{*}{ Tipo de crisis } & \multicolumn{2}{c}{ Primaria } & \multicolumn{2}{c}{ Secundaria } & \multicolumn{2}{c}{ Criptogénica } & \multirow{2}{*}{ Total } \\
\cline { 2 - 6 } & $\mathbf{n}$ & $\mathbf{\%}$ & $\mathbf{n}$ & $\mathbf{( \% )}$ & $\mathbf{n}$ & $\mathbf{( \% )}$ & \\
\hline Generalizadas & 53 & 42,7 & 53 & 42,7 & 18 & 14,5 & 124 \\
Tónico-Clónicas & 22 & 41,5 & 22 & 41,5 & 9 & 17,0 & 53 \\
Tónico & 9 & 34,6 & 13 & 50,0 & 4 & 15,4 & 26 \\
Mioclónica & 9 & 50,0 & 6 & 33,3 & 3 & 16,7 & 18 \\
Clónica & 2 & 18,2 & 8 & 72,7 & 1 & 9,1 & 11 \\
Ausencia & 8 & 72,7 & 2 & 18,2 & 1 & 9,1 & 11 \\
Atónica & 3 & 60,0 & 2 & 40,0 & 0 & 0,0 & 5 \\
& & & & & & & \\
Focal & 18 & 26,1 & 36 & 52,2 & 15 & 21,7 & 69 \\
Compleja & 11 & 31,4 & 16 & 45,7 & 8 & 22,9 & 35 \\
Tónico & 4 & 23,5 & 8 & 47,1 & 5 & 29,4 & 17 \\
Clónica & 3 & 20,0 & 10 & 66,7 & 2 & 13,3 & 15 \\
Mioclónica & 0 & 0,0 & 2 & 100,0 & 0 & 0,0 & 2 \\
\hline
\end{tabular}


$\%(69 / 193)$ de los pacientes experimentaron crisis focales, dentro de ellas, las crisis focales complejas se presentaron en el $50,7 \%(35 / 69)$ y las crisis focales tónicas en $24,6 \%(17 / 69)$.

Las crisis generalizadas fueron igualmente frecuentes de acuerdo a la etiología primaria y secundaria, presentándose ambas en 42,7\% (53/124). Sin embargo, las crisis de tipo mioclónicas y de ausencia pertenecieron en su mayoría al grupo de origen primario. Asimismo, las crisis tónicas y clónicas fueron más frecuentes en la epilepsia secundaria. Por otro lado, en los pacientes con crisis focales, predomino la etiología secundaria en un 52,2\% (36/69) (tabla 3).

La monoterapia fue el esquema de tratamiento más común, en 69,9\% (135/193) de los pacientes, seguido del uso de dos fármacos antiepilépticos en el 22,8\% (44/193). Entre ellos, los fármacos más indicados fueron el ácido valproico y la carbamazepina (tabla 4). El 15\% (29/193) de los pacientes fueron resistentes al tratamiento antiepiléptico, de los cuales el 55,2\% (16/29) padecía epilepsia secundaria.
El $68,4 \%(132 / 193)$ de los casos presentaron al menos una comorbilidad del tipo neurológico o psiquiátrico. Dentro de este grupo, tanto el retraso del desarrollo psicomotor (RDPM), el retardo mental (discapacidad intelectual) (RM) y la parálisis cerebral (PC) fueron las más frecuentes. Estos pacientes pertenecían en su mayoría al grupo de epilepsia secundaria (tabla 5). En los casos de comorbilidad múltiple, la parálisis cerebral y retardo mental fueron prevalentes. Este cuadro se presentó en 16,5\% (15/91) de los pacientes con edad de primera crisis menor de un año y en $12,2 \%(9 / 74)$ del grupo entre 1 a 5 años (tabla 6).

\section{DISCUSIÓN}

La epilepsia en la población infantil presenta diferencias con la población adulta; no obstante, en el Perú esta información aún es escasa. En ese contexto, esta serie de casos brinda una visión general de las características de los niños epilépticos atendidos en un hospital general de Lima.

Tabla 4. Tipo de crisis epiléptica según fármaco utilizado por los niños epilépticos atendidos en el Hospital Cayetano Heredia. Lima, Perú 2010-2016.

\begin{tabular}{|c|c|c|c|c|c|c|c|c|c|c|c|c|}
\hline \multirow{2}{*}{ Tipo de Crisis } & \multicolumn{2}{|c|}{ Ácido valproico } & \multicolumn{2}{|c|}{ Carbamazepina } & \multicolumn{2}{|c|}{ Levetiracetam } & \multicolumn{2}{|c|}{ Clonazepam } & \multicolumn{2}{|c|}{ Lamotrigina } & \multicolumn{2}{|c|}{ Otros } \\
\hline & $\mathbf{n}$ & $\%$ & $\mathbf{n}$ & $\%$ & $\mathbf{n}$ & $\%$ & $\mathbf{n}$ & $\%$ & $\mathbf{n}$ & $\%$ & $\mathbf{n}$ & $\%$ \\
\hline Generalizada & 93 & 79,5 & 25 & 38,5 & 16 & 84,2 & 14 & 82,4 & 11 & 52,4 & 20 & 71,4 \\
\hline Tónica-clónica & 37 & 31,6 & 16 & 24,6 & 6 & 31,6 & 8 & 47,1 & 1 & 4,8 & 6 & 21,4 \\
\hline Tónica & 18 & 15,4 & 5 & 7,7 & 3 & 15,8 & 1 & 5,9 & 3 & 14,3 & 7 & $25,0)$ \\
\hline Mioclónica & 13 & 11,1 & 0 & 0,0 & 6 & 31,6 & 5 & 29,4 & 5 & 23,8 & 4 & $14,3)$ \\
\hline Ausencia & 11 & 9,4 & 0 & 0,0 & 0 & 0,0 & 0 & 0,0 & 2 & 9,5 & 0 & 0,0 \\
\hline Clónica & 10 & 8,6 & 4 & 6,2 & 0 & 0,0 & 0 & 0,0 & 0 & 0,0 & 3 & 10,7 \\
\hline Atónica & 4 & 3,4 & 0 & 0,0 & 1 & 5,2 & 0 & 0,0 & 0 & 0,0 & 0 & 0,0 \\
\hline Focal & 24 & 20,5 & 40 & 61,5 & 3 & 15,8 & 3 & $17,6)$ & 10 & 47,6 & 8 & 28,6 \\
\hline Compleja & 15 & 12,8 & 20 & 30,8 & 1 & 5,2 & 1 & 5,9 & 5 & 23,8 & 1 & 3,6 \\
\hline Tónica & 4 & 3,4 & 11 & 16,9 & 0 & 0,0 & 0 & 0,0 & 5 & 23,8 & 4 & 14,3 \\
\hline Clónica & 4 & 3,4 & 8 & 12,3 & 1 & 5,2 & 1 & 5,9 & 0 & 0,0 & 3 & 10,7 \\
\hline Mioclónica & 1 & 0,9 & 1 & 1,5 & 1 & 5,2 & 1 & 5,9 & 0 & 0,0 & 0 & 0,0 \\
\hline Total & 117 & & 65 & & 19 & & 17 & & 21 & & 28 & \\
\hline
\end{tabular}


Tabla 5. Etiología de la epilepsia según comorbilidad en los niños epilépticos atendidos en el Hospital Cayetano Heredia. Lima, Perú 2010-2016.

\begin{tabular}{|c|c|c|c|c|c|c|c|c|c|c|c|c|c|c|}
\hline \multirow[t]{2}{*}{ Etiología } & \multicolumn{2}{|c|}{$\begin{array}{l}\text { Retraso del } \\
\text { desarrollo } \\
\text { psicomotor }\end{array}$} & \multicolumn{2}{|c|}{$\begin{array}{c}\text { Retardo } \\
\text { mental }\end{array}$} & \multicolumn{2}{|c|}{$\begin{array}{l}\text { Parálisis } \\
\text { cerebral }\end{array}$} & \multicolumn{2}{|c|}{$\begin{array}{l}\text { Trastorno de } \\
\text { la conducta }\end{array}$} & \multicolumn{2}{|c|}{$\begin{array}{c}\text { Trastorno } \\
\text { de déficit de } \\
\text { atención e } \\
\text { hiperactividad }\end{array}$} & \multicolumn{2}{|c|}{$\begin{array}{l}\text { Trastorno } \\
\text { del espectro } \\
\text { autista }\end{array}$} & \multicolumn{2}{|c|}{ Otros } \\
\hline & $\mathbf{n}$ & $\%$ & $\mathbf{n}$ & $\%$ & $\mathbf{n}$ & $\%$ & $\mathbf{n}$ & $\%$ & $\mathbf{n}$ & $\%$ & $\mathbf{n}$ & $\%)$ & $\mathbf{n}$ & $\%$ \\
\hline Primaria & 16 & 26,2 & 14 & 25,9 & 7 & 14,0 & 5 & 38,5 & 3 & 33,3 & 3 & 37,5 & 3 & 18,8 \\
\hline Secundaria & 34 & 55,7 & 29 & 53,7 & 37 & 74,0 & 6 & 46,1 & 4 & 44,4 & 3 & 37,5 & 9 & 56,2 \\
\hline Criptogénica & 11 & 18,0 & 11 & 20,4 & 6 & 12,0 & 2 & 15,4 & 2 & 22,2 & 2 & 25,0 & 4 & 25,0 \\
\hline Total & 61 & & 54 & & 50 & & 13 & & 9 & & 8 & & 16 & \\
\hline
\end{tabular}

Tabla 6. Comorbilidad según edad de primera crisis en los niños epilépticos atendidos en el Hospital Cayetano Heredia. Lima, Perú 2010-2016.

\begin{tabular}{|c|c|c|c|c|c|c|c|c|}
\hline \multirow[t]{2}{*}{ Comorbilidad } & \multicolumn{2}{|c|}{$\begin{array}{l}\text { Niños con } \\
\text { comorbilidad }\end{array}$} & \multicolumn{2}{|c|}{$<1$ año } & \multicolumn{2}{|c|}{$1-5$ años } & \multicolumn{2}{|c|}{ 6-14 años } \\
\hline & $\mathbf{n}$ & $\%$ & $\mathbf{n}$ & $\%$ & $\mathbf{n}$ & $\%$ & $\mathbf{n}$ & $\%$ \\
\hline Retraso del desarrollo psicomotor & 61 & 46,2 & 39 & 42,9 & 19 & 25,7 & 3 & 10,7 \\
\hline Retardo mental & 54 & 40,9 & 23 & 25,3 & 22 & 29,7 & 9 & 32,1 \\
\hline Parálisis cerebral & 50 & 37,9 & 36 & 39,6 & 12 & 16,2 & 2 & 7,1 \\
\hline Trastorno de la conducta & 13 & 9,8 & 4 & 4,4 & 7 & 9,5 & 2 & 7,1 \\
\hline Parálisis cerebral + Retardo mental & 26 & 19,7 & 15 & 16,5 & 9 & 12,2 & 2 & 7,1 \\
\hline Retraso del desarrollo psicomotor + Parálisis cerebral & 20 & 15,2 & 15 & 16,5 & 5 & 6,8 & 0 & 0,0 \\
\hline Retraso del desarrollo psicomotor + Retardo mental & 10 & 7,6 & 4 & 4,4 & 4 & 5,4 & 2 & 7,1 \\
\hline Otros & 33 & 25,0 & 16 & 17,6 & 10 & 13,5 & 7 & 25,0 \\
\hline Total & 132 & & 91 & & 74 & & 28 & \\
\hline
\end{tabular}

No se encontró diferencia con respecto a la distribución según género en comparación con otros estudios; sin embargo, la epilepsia fue predominante en la población masculina, observación que posiblemente se relaciona con los factores genéticos y mayor exposición de los niños a diversos factores lesivos al sistema nervioso central (SNC) $(12,13)$.

Casi la mitad de los pacientes de esta serie iniciaron las crisis epilépticas antes del año de edad. Como se conoce, la edad de la primera crisis es un factor importante para el diagnóstico y subsecuente evolución clínica. Además, el inicio precoz podría estar asociado con incremento de déficits neurológicos, menor control de las crisis y la muerte $(3,14)$. Si bien la mayoría de los pacientes epilépticos inician sus crisis antes de los 5 años, se han encontrado diferencias en la edad de presentación (15). En España, Ramos y col. encontraron que cerca del $50 \%$ de pacientes presentaron su primera crisis epiléptica entre los 4 y 9 años (16). En un estudio más reciente en Cuba, la mitad de los pacientes iniciaron con las crisis entre 1 y 4 años de edad (7). Sin embargo, diversos autores reportan una incidencia máxima de epilepsia en el primer año de vida para después descender conforme se avanza hacia la adolescencia (17).

Un hallazgo especial fue el predominio de crisis generalizadas, muy semejante a un estudio chileno en pacientes de 5 meses a 19 años (18). No obstante, la mayoría de autores reportan que las crisis focales son más frecuentes en los niños (17). Estas diferencias pueden explicarse por que muchas veces no se conoce el inicio de las crisis, o que éstas se originan en áreas mediales del cerebro.

La etiología secundaria fue la más frecuente, destacando las malformaciones cerebrales, los eventos adversos perinatales y las infecciones del sistema nervioso central. A pesar de las investigaciones, se sabe muy poco sobre la etiología de la epilepsia en la población pediátrica. Larson y col. en un estudio poblacional en Suiza, encontraron que las epilepsias primarias se presentaban en un $40,4 \%$, similar a 
otros estudios españoles y chilenos $(5,16,18)$. En los últimos años la video electroencefalografía ha sido de gran ayuda para aclarar la lateralización de las crisis (19). Camfield reportó que al menos el $50 \%$ de las epilepsias son de causa desconocida y un tercio son de causa estructural o metabólica. Además, refiere que es difícil conocer el verdadero origen de esta enfermedad, y que las clasificaciones han ido cambiando con el tiempo (20). En general la epilepsia secundaria es más frecuente en países de bajos recursos y generalmente sus causas son prevenibles $((21,22)$.

Los estudios muestran mayor incidencia de epilepsias secundarias durante los primeros 3 años de vida $(16,23)$. En esta serie las malformaciones cerebrales y los eventos perinatales fueron las causas predominantes, hallazgo que está en relación con el mayor uso de neuroimagen en los últimos años (24) y con la frecuencia de factores de riesgo perinatales no controlados (21). Hacemos notar que en este estudio no se incluyeron pacientes sin neuroimagen, factor por el cual se excluyeron cuatro casos que se podrían haberse definido sólo por el patrón electroencefalográfico.

La etiología primaria fue predominante en los niños que iniciaron sus crisis después del año de edad. Dos estudios españoles realizados en una población escolar mencionan que entre $40,8 \%$ y $57,4 \%$ de las epilepsias fueron de origen idiopático. Las epilepsias criptogénicas se mantuvieron estables a lo largo de toda la infancia y en menor proporción se encontraron las de etiología secundaria $(16,23)$, mostrando un comportamiento similar a nuestra población.

Referente a la epilepsia secundaria en mayores de un año, es importante mencionar que en el grupo de 1 a 5 años de edad resultó frecuente la presencia de síndromes neurocutáneos. Al respecto, se conoce que el $80 \%$ de pacientes con esta patología son diagnosticados antes de los 3 años de edad y hasta un $70 \%$ son generalmente refractarios a tratamiento (25). En los niños mayores de 5 años, el traumatismo encefalocraneano (TEC) es una de las causas más frecuentes $(17,26,27)$. Un estudio realizado en Chile menciona que la frecuencia podría ser hasta $14 \%$ de pacientes epilépticos menores de 14 años (28). En un trabajo publicado en Perú en el 2002, se evaluaron los principales factores etiológicos en niños diagnosticados con epilepsia secundaria, donde el TEC ocupó el cuarto lugar (29).

Sorprendentemente no se encontraron pacientes con epilepsia por neurocisticercosis. Como se conoce, esta enfermedad tiene alta prevalencia en el Perú $(7,1 \%$ a $26,9 \%)$ y por lo general los pacientes inician con crisis focales aisladas (22). Un reporte realizado en la misma institución ( $\mathrm{HNCH})$ entre los años de 1998-1999 encontró que la principal causa de crisis focales en niños fue la neurocisticercosis en el 39\% de los casos (30). Sin embargo, cabe destacar que en ese estudio prospectivo se examinaron pacientes atendidos en emergencia y hospitalización. Sin embargo, es evidente que ha disminuido la neurocisticercosis como causa de crisis en nuestro hospital, no obstante, existe la posibilidad que algunos niños no hayan continuado con el seguimiento neurológico después de la atención hospitalaria.

El fármaco más utilizado en las crisis generalizadas fue el ácido valproico y en las crisis focales fue la carbamazepina. En un estudio transversal realizado por Liu et al encontraron que el uso de ácido valproico y carbamazepina ha disminuido con el transcurso de los años, Por lo contrario, el uso de oxcarbazepina y levetiracetam se ha incrementado de manera significativa, siendo el levetiracetam el fármaco más utilizado durante los años 2008 y 2009 (31). Para las crisis focales, los estudios mencionan que el ácido valproico tiene una eficacia similar o levemente inferior a la carbamazepina siendo estos dos fármacos el tratamiento de elección $(32,33)$.

Se conoce desde antes que $50 \%$ de los pacientes controlan sus crisis con el primer fármaco antiepiléptico y que la adición de un segundo fármaco eleva la probabilidad a $75 \%$; no obstante, hasta un $25-30 \%$ de los pacientes pueden ser refractarios al tratamiento (3). En esta serie, el $15 \%$ de casos fueron refractarios al tratamiento, los cuales podrían ser candidatos a tratamiento no farmacológico. Al respecto, Campos y col. estimaron que cerca de 650 niños por año serían tributarios de cirugía de epilepsia en nuestro país, entre las que destacan las malformaciones cerebrales $(34,35)$.

La comorbilidad es un aspecto muy importante. En un estudio chileno realizado con 239 niños epilépticos, se encontró en $80 \%$ de su población (18). Similar a lo encontrado en esta serie de casos, donde cerca del $70 \%$ de los pacientes presentó al menos una comorbilidad neuropsiquiátrica. Asimismo, Castaño encontró en una población colombiana que el RDPM y RM se presentaban frecuentemente de manera conjunta (15). En un estudio chileno que comparó la presencia de comorbilidades en epilepsias, concluyó que las de origen secundario tienen mayor probabilidad (18). Estas características concuerdan con nuestra serie, 
pues más del $50 \%$ de las comorbilidades se presentan en los pacientes con epilepsia secundaria.

La relevancia de estos hallazgos se relaciona con dos hechos fundamentales, primero tener en cuenta que en nuestro medio es muy importante sospechar en crisis epilépticas en niños pequeños con trastornos paroxísticos. Y segundo, que, frente a la gran causalidad secundaria, es muy importante que nuestro sistema salud organice campañas de prevención para mejorar la atención perinatal, para educar sobre los efectos tóxicos de sustancias usadas de manera popular, para disminuir los accidentes del hogar y para incluir en los Programas de Estudios de Medicina los conceptos sobre epilepsia en niños.

Entre las limitaciones del estudio, se observa la dificultad para obtener un registro detallado de las crisis epilépticas y la pérdida de pacientes por datos incompletos. Sin embargo, entre las fortalezas destaca por ser una serie de casos de 7 años consecutivos de observación en un Servicio Neuropediátrico con criterios clínicos uniformes.

En conclusión, la mayoría de los niños iniciaron su primera crisis antes del año de edad, generalmente de causa secundaria, siendo frecuentes las malformaciones cerebrales y los eventos perinatales. En los niños mayores prevaleció la etiología primaria. Esta serie de casos muestra un predominio de las crisis generalizadas y de pacientes frecuentemente controlados con monoterapia, siendo las drogas más utilizadas el ácido valproico y la carbamazepina. Asimismo, destaca la alta frecuencia de comorbilidad, como retraso del desarrollo psicomotor, parálisis cerebral y retardo mental.

Agradecimientos. Al Departamento de Estadística del HNCH y al Dr. Daniel Guillén Mendoza por sus aportes al análisis y presentación del estudio.

\section{Declaración de Financiamiento: Proyecto}

autofinanciado.

Los autores declaran no tener conflicto de interés.

\section{Correspondencia:}

Dr. Daniel Guillén Pinto

Av. Honorio Delgado 430 Urb. Ingeniería, San

Martín de Porres,

Código Postal Lima 31. Lima, Perú.

Correo electrónico: dguillenpinto@gmail.com

\section{REFERENCIAS BIBLIOGRÁFICAS}

1. Fisher RS, Van Emde Boas W, Blume W, Elger C, Genton P, Lee P, et al. Epileptic seizures and epilepsy: definitions proposed by the international league against epilepsy (ILAE) and the international bureau for epilepsy (IBE). Epilepsia. 2005; 46(4): 470-472.

2. Wolf SM, Engel P. Recognition and management of pediatric seizures. Pediatr Ann. 2006; 35(5): 332-344.

3. Campos M, Kanner A. Epilepsias: Diagnóstico y tratamiento. Santiago de Chile: Mediterráneo; 2004.

4. Medina C. Epilepsia: Aspectos clínicos y psicosociales. Bogotá: Médica Panamericana; 2004.

5. Larsson K, Eeg-Olofsson O. A population based study of epilepsy in children from a Swedish county. Eur J Paediatr Neurol. 2006; 10(3):107-113.

6. Díaz D, Conde Y, Conde M, Navia I, Conde B. Epidemiología clínica de la epilepsia en niños y adolescentes del municipio Sancti Spíritus. Gac Med Espirit. 2013; 15(2): 154-163.

7. Martínez X, Duarte Y, Portales E, Mirabal G. Estudio epidemiológico de la epilepsia infantil en el municipio de Bahía Honda. Rev Ciencias Médicas. 2011; 15(1): 76-88.

8. Duchowny M, Harvey A. Pediatric epilepsy syndromes: An update and critical review. Epilepsia. 1996; 37(1): S26-S40.

9. Wilfong A. Seizures and epilepsy: Classification, etiology and clinical features. Massachusetts, Estados Unidos: UpToDate; 2016. (Fecha de acceso 19 de agosto del 2018) Disponible en: https://www. uptodate.com/contents/seizures-and-epilepsy-inchildren-classification-etiology-and-clinical-features

10. Tanriverdi M, Mutluay F, Tarakci D, Güler S, Iscan A. The impact of epilepsy on preschool children and their families. Epilepsy Behav. 2016; 62: 6-11.

11. Berg A, Berkovic S, Brodie M, Buchhalter J, Cross $\mathrm{J}$, Van Emde Boas W, et al. Revised terminology and concepts for organization of seizures and epilepsies: Report of the ILAE Commission on Classification and Terminology, 2005-2009. Epilepsia. 2010; 51(4): 676-685.

12. Sun Y, Vestergaard M, Pedersen C, Christensen J, Olsen J. Apgar scores and long-term risk of epilepsy. Epidemiology. 2006; 17(3): 296-301.

13. Alcázar J, Castaño J, Fragoso L, García S, Giraldo $\mathrm{S}$, Jiménez $\mathrm{S}$, et al. Factores de riesgo presentes en una muestra de población epiléptica de Manizales, Caldas, Colombia. Arch Med. 2008; 8(1): 32-39.

14. Appleton R, Gibbs J. Epilepsy in Childhood and Adolescence. New York: Martin Dunitz; 2003.

15. Castaño J, Campos Y, Duque C, Grisales E, Mera T, Rodríguez $\mathrm{G}$, et al. Caracterización de la población con epilepsia atendida en un hospital infantil en Manizales 2013-2014. CES Medicina. 2016; 30(2): 139-147. 
16. Ramos J, Carrasco Ll, Vázquez M, Calvo MD, Cassinello E. Epidemiología de la epilepsia en la edad pediátrica: Tipos de crisis epilépticas y síndromes epilépticos. An Esp Pediatr. 1996; 45(3): 256-260.

17. Cowan L. The epidemiology of the epilepsies in children. Ment Retard Dev Disabil Res Rev. 2002; 8(3): 171-181.

18. Jeldres E, Devilat M, Peralta S, Gómez V. Comorbilidad en niños con epilepsia. Rev Chil Epilepsia. 2010; 10(2):19-28.

19. Aguilar L, Rodríguez R, Morgade R, Valdivia I, García R, Bonet E. Experiencia en el uso del videoelectroencefalograma en el servicio de neurofisiología clínica. Rev Cubana Pediatr. 2012; 84(3): 234-243.

20. Camfield P, Camfield C. Incidence, prevalence and aetiology of seizures and epilepsy in children. Epileptic Disord. 2015; 17(2): 117-123.

21. Medina M, Rivera F, Tori A, Montiel J, Guillén D. Frecuencia, características y mortalidad asociada a la enfermedad neurológica en la Unidad de Cuidados Intensivos Neonatales del Hospital Nacional Cayetano Heredia. Rev Peru Pediatr. 2007; 60(1): 1119.

22. García H, Gonzales A, Rodriguez S, Gonzalvez G, Llanos-Zavalaga F, Tsang V, et al. Epidemiología y control de la cisticersosis en el Perú. Rev Peru Med Exp Salud Publica. 2010; 27(4): 592-597.

23. Durá T, Yoldi M, Gallinas F. Incidencia de la epilepsia infantil. An Pediatr. 2007; 67(1): 37-43.

24. Prats J, Garaizar C, Gil J, Pérez J. Frecuencia y diversidad clínica actual de las disgenesias corticales cerebrales. An Esp Pediatr. 1998; 48(5): 499-504.

25. Stafstrom C, Staedtke V, Comi A. Epilepsy Mechanisms in Neurocutaneous Disorders: Tuberous Sclerosis Complex, Neurofibromatosis Type 1, and Sturge-Weber Syndrome. Front Neurol. 2017; 8(87): $1-14$.
26. Hauser W, Annegers J, Kurland L. Incidence of epilepsy and unprovoked seizures in Rochester, Minnesota: 1935-1984. Epilepsia. 1993; 34(3): 453458.

27. Guillén-Pinto D, Zea A, Guillén-Mendoza D, Situ M, Reynoso C, Milla L, et al. Traumatismo encefalocraneano en niños atendidos en un hospital nacional de Lima, Perú 2004-2011. Rev Peru Med Exp Salud Publica. 2013; 30(4): 630-634.

28. Braga P. Epilepsia post traumática. Rev Chil Epilepsia. 2012; 12(2): 85-92.

29. Rodríguez L, Quispe Y, Sifuentes J. Factores de riesgo de epilepsia secundaria en niños. Rev Neuropsiquiatr. 2002; 65(2):136-141.

30. Gaffo A, Guillen D, Campos P, Burneo J. Cisticercosis como causa principal de crisis parciales en niños en Perú. Rev Neurol. 2004; 39(10): 924-926.

31. Liu X, Carney P, Bussing R, Segal R, Cottler L, Winterstein A. Trends in Antiepileptic Drug Use in Children and Adolescents with Epilepsy. Pediatr Neurol. 2017; 74: 32-40.

32. Pozo A, Pozo D. Tratamiento con medicamentos antiepilépticos en el niño. Rev Cubana Pediatr. 2013; 85(4):497-516.

33. Tomson T, Battino D, Perucca E. Valproic acid after five decades of use in epilepsy: time to reconsider the indications of a time-honoured drug. Lancet Neurol. 2016; 15(2): 210-218.

34. Campos M, Ríos L, Solari F, Varela X, Otayza F. Consideraciones quirúrgicas propias de la epilepsia en niños, comentarios sobre Perú. Rev Neuropsiquiatr. 2015; 78(3): 130-139.

35. Harvey A, Cross J, Shinnar S, Mathern G. Defining the spectrum of international practice in pediatric epilepsy surgery patients. Epilepsia. 2008; 49(1): 146-155.

Recibido: 20/08/2018

Aceptado: 28/09/2018 